\title{
Abdominal pain in the elderly: mini review
}

\begin{abstract}
Abdominal pain is a common complaint in the ED, in the elderly it can mean nothing or it can be a surgical emergency, recognition of the etiology and appropriate treatment can be live saving for a patient that not always can tell us what is going on.
\end{abstract}

Volume 3 Issue 4 - 2018

\author{
Ernesto Arriaga Morales \\ Department of emergency medicine specialist, Hospital Angeles \\ Lindavista, Mexico
}

Correspondence: Ernesto Arriaga-Morales, Department of emergency medicine specialist, Hospital Angeles Lindavista, Mexico,Tel 0445538802060,Email earriaga_2@hotmail.com

Received: June 27, 2018 | Published: July 06, 2018

\section{Introduction}

Abdominal pain is a common complaint in the emergency department, ${ }^{1,2}$ from pediatric to geriatric patients, both have unique characteristics, but share a common situation, a communication bias; for the elderly patients is difficult to tell us their complaints, the characteristics of their pain, when does the problem begun. Worst of all, sometimes they suffer delirium ${ }^{3}$ and it's impossible for them to tell the physician if they have urinary discomfort or inferior right quadrant abdominal pain. ${ }^{4}$

\section{Presentation: history and diagnostic test}

When we make the clinical history of our elderly patient we should try to get as much information as we can including, in the best scenario: time of onset of the pain, sudden versus gradual onset, location, quality and severity of pain, if the pain has radiation, the factors that precipitate, aggravate or palliate pain, history of previous similar episodes, ability to pass stool of flatus; associated symptoms like fever, (Since 2008 the Infectious Disease Society of America proposed a new definition for fever in older adults, and is defined as follows: A single oral temperature greater than $100^{\circ} \mathrm{F}\left(>37.8^{\circ} \mathrm{C}\right)$; or Repeated oral temperatures greater than $99^{\circ} \mathrm{F}\left(>37.2^{\circ} \mathrm{C}\right)$; or Repeated rectal temperatures greater than $99.5^{\circ} \mathrm{F}\left(>37.5^{\circ} \mathrm{C}\right)$; or Increase in temperature of greater than $2^{\circ} \mathrm{F}\left(>1.1^{\circ} \mathrm{C}\right)$ over baseline temperature $)^{5}$ urinary symptoms, hyporexia or anorexia, nausea, vomiting or diarrhea and the characteristics of the last one cause it can be even melena or blood in the stool; it is important to remember to question non abdominal symptoms like dyspnea or chest pain cause the atypical presentation of myocardial infarction is not that atypical in the elderly. ${ }^{2,3}$ We have to consider all the comorbidities of our patient like diabetes or cardiovascular disease, smoking, alcohol use and NSAID use. ${ }^{6}$

Medical history can approach us to the possible etiology of the pain, but not all of the elderly patients can help us with that, and we have to rely more on physical examination and laboratory or imaging techniques. ${ }^{1,4}$ During physical examination, we have to give special attention to vital signs, and not to forget pulmonary and cardiovascular systems, for example in the presence of abdominal pain and atrial fibrillation it is likely the diagnosis of mesenteric ischemia. $^{7}$ Auscultation can add important information to our differential, for example high-pitched bowel sound are associated with bowel obstruction. On the other hand, absent bowel sounds may indicate adynamic ileus or advanced bowel obstruction. It will be key to remember that elderly patients with peritonitis may lack classic peritoneal signs. ${ }^{1}$ Palpable mass can be either a chronic condition like a malignancy or sign of and acute situation like a phlegmon from ruptured appendix. A pulsatile mass should be examined to rule out an abdominal aneurism. ${ }^{2}$ In the elderly rectal examination to identify fecal impaction, that is a common situation in the elderly. ${ }^{8}$

In and classic study, with 9585 patients with elderly patients with peritonitis they found a diagnostic accuracy of only $47 \%$. Wroblewski proposed as the possible explanation of the low level of diagnostic accuracy may be the absence of symptoms and signs for example even abdominal pain occurred in only $50 \%$. A probable explanation is the decrease of pain sensitivity in the skin and gut associated with the age. This study reports that no symptom has a sensitivity above 0.48 , while the combination of abdominal pain, nausea, vomiting and tachycardia have a specificity of 0.93 and in the absence of abdominal pain the combination of nausea, vomiting, fever and tachycardia have a specificity of $0.88 .^{9}$

Diagnostic test should include blood chemistry including full electrolyte panel to rule out metabolic ileus, complete blood count. Imaging test are key to diagnosis, maintain a low threshold to order them and choose the technique guided by the suspected diagnosis (for example: ultrasound for biliary tract pathology and computed tomography for bowel obstruction). ${ }^{4,10}$

\section{Treatment in the ED: observation vs surgery}

Some of our elderly patients with abdominal pain will only be suffering of constipation, urinary tract infection or other mild diseases, but some of them will require emergent surgery for example those with mesenteric ischemia or appendicitis. The first step will always be assessing airway, breathing, circulation and vital signs. Evaluating the severity of the disease with an appropriate history, physical examination, diagnostic workup and early consultation with surgery or geriatric specialist are necessary to provide appropriate treatment and improve outcomes. Some patients need monitor or intravenous (IV) lines, but we have to remember that not everybody needs and intravenous, and in the elderly some patient can develop delirium when they are invaded with an IV or a Foley catheter (incontinence is not an indication to place it). Keep every patient with abdominal pain with nothing by mouth until surgical pathology is excluded, but begin foods as soon as possible if surgery need is ruled out. Abdominal sepsis should be managed according the updated sepsis guidelines, 
but remembering the particularities of our patient group, the aging of every organ (heart, lungs, kidney and liver for example) force us to be careful when calculating doses. Fluid reanimation, directed antibiotic administration in the first twenty minutes, source control in the surgical patient, urine output and cardiac monitoring according with the needing of our patients (invasive or non-invasive). ${ }^{11}$

\section{Fluid reanimation}

Fluids are ubiquitous in the modern emergency room, but appropriate amount of fluids in a difficult art, too much can cause glycocalyx alteration and fluid leakage to the lung, abdominal compartment of the subcutaneous tissue. Remember elderly patients can suffer congestive heart failure or chronic kidney disease more frequently than any other patient group. And that too little can slow down the recovery of our patients, today the best approach is ultrasound guided fluid reanimation, inferior cava vein diameter, carotid artery peak velocity variation and cardiac output serial measurements. The last one is the newer technique which gives us information about how much fluids can tolerate our patient and if the heath can handle it, but a combination of techniques can tell us enough information of our patient to drive him safe to the recovery.

\section{Pain and palliative care}

Pain management is a universal right, then every patient young or old have to receive appropriate analgesia, in the elderly we have to consider the risk of gastrointestinal tract bleeding with NSAIDs and the risk of hypotension with opioids, but with a carefully evaluation we can achieve enough pain relieve and minimal or none adverse effects, in undifferentiated abdominal pain small doses of opioids is recommendable. ${ }^{12}$ Family support, exposure to sun light, no unnecessary invasive procedures, social work, psychology and thanatology interventions are ways to support our elderly patient when visiting the ED. ${ }^{13}$

\section{Discharge or admit?}

Admit every patient with surgical pathology, with hypotension, altered mental status, severe pain, persistent tachycardia, consider intensive care unit for close monitoring. ${ }^{6,14}$ If you decide to discharge an elderly patient with abdominal pain, be careful, discharging them should be the last option.

\section{Acknowledgements}

I want to thank to my family for their support, specially to my wife and my next to come son. Also I want to thank to the people who drove me in to the emergency medicine, to all these colleagues thank you for making me find my happy place.

\section{Conflict of interest}

The author declares no conflict of interest.

\section{References}

1. Ragsdale L, Southerland L. Acute abdominal pain in the older adult. Emerg Med Clin North Am. 2011;29(2):429-48.

2. SS Mehta. Abdominal pain in the elderly. Medicine Update. 2008;18:239 45 .

3. Spangler R, Pan TV, Khoujah D, et al. Abdominal emergencies. Int J Emerg Med. 2014;200-19.

4. Chang CC, Wang SS. Acute abdominal pain in the elderly. International Journal of Gerontology. 2007;1(2):77-82.

5. High KP, Bradley SF, Gravenstein S, et al. Clinical practice guideline for the evaluation of fever and infection in older adult residents of long-term care facilities: 2008 update by the infectious diseases society of america. Clin Infect Dis. 2009;48(2):149-71.

6. Corey L, Dawyne C. Diagnosis of acute abdominal pain in older patients. Am Fam Physician. 2006;74(9):1537-44.

7. Hunt SJ, Coakley FV, Webb EM, et al. Computed tomography of the acute abdomen in patients with atrial fibrillation. J Comput Assist Tomogr. 2009;33(2):280-5.

8. Williams ME. The geriatric abdominal and rectal examination overview and general inspection of the abdomen. Medscape. 2012.

9. Wroblewski M, Mikulowski P, Peritonitis in geriatric inpatients, Age Ageing. 1991.20(2):90-94.

10. Reginelli A, Russo A, Pinto A, et al. The role of computed tomography in the preoperative assessment of gastrointestinal causes of acute abdomen in elderly patients. Int J Surg. 2014;12:S181-S186.

11. Rowe TA, McKoy JM. Sepsis in older adults. Infect Dis Clin N Am. 2017;31(4):731-742.

12. Pasero C. Acute Abdominal Pain: manage without delay. Medscape. 2012.

13. Gower L, Gatewood M, Kang C. Emergency department management of delirium in the elderly. West J Emerg Med. 2012;13(2):194-201.

14. Jensen JS, Ipsen HL, Jørsboe H. High use of antibiotics in elderly patients at discharge after hospitalization for acute abdominal pain. Scand J Trauma Resusc Emerg Med. 2015;23(Suppl 1):A25 